\title{
Order Selection of Spatial and Temporal Autoregressive Models with Errors in Variables
}

\author{
Mauro Coli, Lara Fontanella and Luigi Ippoliti \\ Department of Quantitative Method and Economic Theory, University “G. D’Annunzio”, Chieti, Italy
}

\begin{abstract}
In this paper we consider the issues involved in model order selection for processes observed with additive Gaussian noise. In particular, we discuss conditional maximum likelihood estimation of noisy autoregressive models and provide an estimator that takes care of the observational noise. The estimator is weakly consistent, can be computed in only $O(n)$ steps and can be used in the automatic model identification phase. Using information criteria, an extensive simulation study shows the results of order selection in the context of time and spatial series analysis.
\end{abstract}

Keywords: autoregressive processes, information criteria, conditional maximum likelihood, image analysis.

\section{Introduction}

Inference on temporal, spatial and spatio-temporal autoregressive models, are usually carried out conditionally on a previously selected lag order [5], [6]. In many cases the lag order selection is carried out using several information criteria which have relative advantages depending upon the situation in which they are used. There has been considerable development during the last decade on the question of time series model selection. For a review see for example [4]. On the other hand, the literature on spatial model selection is sparse and exceptions are the papers [11] and [16]. In this article we are concerned with the model order selection problem of a temporal or spatial autoregressive (AR) process $X$ that is not observed directly. Instead, we assume the analyst observes the process $Y$ such that

$$
Y=X+\eta
$$

where $\eta$ is a white noise with variance $\sigma_{\eta}^{2}$, independent of $X$. Although at a first sight it could appear restrictive, the hypothesis that $X$ may be represented by AR models permits to overcome the computational burden which is usually encountered with huge data sets or when a movingaverage (MA) component is considered in the model. In fact, apart from the univariate case where the convergence of the maximum likelihood function may not be attained at all [18], it is known that in the multivariate case, in addition to requiring causality and invertibility, the consideration of the MA component needs further assumptions which may suggest to fit only vector AR models [17]. Thus, all the aforementioned problems might partially explain why there have been only a few accounts in the literature of studies involving ARMA or VARMA order selection procedures.

To give insights into the importance of how the signal parameters estimation might be affected by an additive Gaussian error we present in the context of time series analysis, a simple example regarding a zero mean $\mathrm{AR}(1)$ process [5]. In particular, to give a flavour of types of behaviour of the Ordinary Least Square (OLS) estimator, Table 1 shows the results of a set of simulations on 500 samples generated with parameters $n=200, \phi=0.3,0.5,0.8, \sigma_{\eta}^{2}=0.4$, $0.6,1$ and $\sigma_{\varepsilon}^{2}=1$; where $n$ is the sample size, $\phi$ is the autoregressive parameter and $\sigma_{\varepsilon}^{2}$ the variance of the driving noise.

As expected, results highlight a strong bias which is more evident particularly when the signal-to-noise-ratio (ratio between signal and 


\begin{tabular}{|c|c|c|c|}
\cline { 2 - 4 } \multicolumn{1}{c|}{} & \multicolumn{3}{c|}{ Real Parameters } \\
\cline { 2 - 4 } \multicolumn{1}{c|}{} & $\phi=0.8$ & $\phi=0.5$ & $\phi=0.3$ \\
\hline \multirow{2}{*}{$\sigma_{\eta}^{2}=0.4$} & 0.6725 & 0.4284 & 0.2572 \\
& $(0.0655)$ & $(0.0656)$ & $(0.0675)$ \\
\hline \multirow{2}{*}{$\sigma_{\eta}^{2}=0.6$} & 0.5760 & 0.3634 & 0.2170 \\
& $(0.0709)$ & $(0.0709)$ & $(0.0732)$ \\
\hline \multirow{2}{*}{$\sigma_{\eta}^{2}=1$} & 0.3902 & 0.2441 & 0.1520 \\
& $(0.0871)$ & $(0.0767)$ & $(0.0702)$ \\
\hline
\end{tabular}

Table 1. The means and standard errors (in brackets) of the OLS estimates of an AR(1)+Noise model.

noise variances, SNR) decreases. However, such a result is not surprising since the introduction of the observational noise affects the correlation structure of the original process. This can be easily shown for the AR(1)+Noise model. In fact, since $\mathrm{Y}$ is the sum of two independent stationary components, we have that

$$
\gamma_{y}(h)=\left\{\begin{array}{cc}
\frac{\sigma_{\varepsilon}^{2}}{1-\phi^{2}}+\sigma_{\eta}^{2} & \text { for } h=0 \\
\left(\frac{\phi^{h}}{1-\phi^{2}}\right) \sigma_{\varepsilon}^{2} & \text { for } h>0
\end{array}\right.
$$

where $\gamma_{y}(h)=\operatorname{Cov}\left[Y_{t}, Y_{t-h}\right], h$ is the temporal lag and $t$ the discrete index of times. Consequently, the autocorrelation function of the observed process is

$$
\rho_{y}(h)=\frac{\gamma_{y}(h)}{\gamma_{y}(0)}=\left(1+\frac{\sigma_{\eta}^{2}}{\sigma_{\varepsilon}^{2}}\left(1-\phi^{2}\right)\right)^{-1} \phi^{h}
$$

that shows that the process $Y_{t}$ is not $\mathrm{AR}(1)$ unless $\sigma_{\eta}^{2}=0$.

Actually, the addition of white noise to an $\operatorname{ARMA}(m, q)$ process was discussed in [5]. In general, if $m>q$, and the process is observed with error, the resulting observed process is an $\operatorname{ARMA}(m, m)$ process. The resulting $2 m$ parameters are a function of the original $m+q$ parameters plus the variance of the observational noise. A point worth noting is that the inclusion of the observational error is sometimes related to the opportunity to find a more parsimonious model than simply fitting ARMA processes. This is particularly evident for the autoregressive case. In fact, provided the underlying process $X_{t}$ is pure autoregressive $\operatorname{AR}(m)-$, it is only necessary to estimate $m+1$ parameters rather than $2 m$ parameters for an $\operatorname{ARMA}(m, m)$ process.
A natural way of estimating the $m+1$ parameters is via Kalman filter [12] that directly allows to take into account the presence of a measurement noise. However, out of the state space form, as we have seen in Table 1, a direct application of the OLS estimator leads to biased results which alter the one-step ahead prediction error variance.

The purpose of this paper is to propose an OLS estimator that, taking into account the presence of the observational error, can be used as a quick tool for autoregressive model order selection. The paper is outlined as follows. In Section 2 we present the spatial and temporal autoregressive models as well as the Adjusted Least Square estimator $(A L S E)$ that takes into account the presence of an external source of error. The problem of model identification involving order selection and the information criteria used in the paper are described in Section 3. Performance of the methods discussed in Sections 2 and 3 is the principal subject of a simulation exercise, the design of which is the main component of Section 4. Finally, in Section 5 we conclude the paper with a discussion.

\section{Models and Conditional Maximum Likelihood Estimation}

In the framework of temporal and spatial analysis, we investigate two different models.

\subsection{Temporal Autoregressive Models}

In this case we consider a zero-mean autoregressive process of order $m[5]$, given by

$$
\sum_{j=0}^{m} \phi_{j} X_{t-j}=\varepsilon_{t}, \quad \phi_{0}=1
$$

where $m$ is unknown, $\phi_{j}, j=1, \ldots, m$, are real parameters such that all the roots of the polynomial

$$
\sum_{j=0}^{m} \phi_{j} z^{j}=0
$$

lie outside the unit circle, and $\varepsilon_{t}$ is a Gaussian white noise process with variance $\sigma_{\varepsilon}^{2}$. This implies that there exists a true value of $m$, denoted by $m_{0}$ such that $\phi_{j}(m)=0$ for $m>m_{0}$. In 
this context, several information criteria may be used to pick the lag order of the model.

\subsection{Gauss Markov Random Fields}

They are widely used as models in spatial analysis of lattice data [6], [9], [7]. Because of the strong association to image analysis we shall mainly think of the spatial sites as pixels of a $(R \times C)$ lattice. Thus, we say that the grey levels are distributed according to a zero-mean Gauss Markov Random Field (GMRF) if the distribution of $X$ is multivariate normal with conditional means and conditional variances

$$
\begin{gathered}
E\left(X_{i} \mid X_{j}: j \in \delta_{i}\right)=\sum_{j \in \delta_{i}} \beta_{i, j} x_{j} \\
\operatorname{Var}\left(X_{i} \mid X_{j}: j \in \delta_{i}\right)=\tau^{2}
\end{gathered}
$$

where $\delta_{i}$ is the set of neighbors of pixel $i$ (not including $i)$. Here $x=\left(x_{1}, x_{2}, \ldots, x_{n}\right)^{\mathrm{T}}$ is a $n$-vector of grey-levels at pixel $(i, j),(i=$ $1, \ldots, R ; j=1, \ldots, C)$. Note that $n=(R \times C)$ and that the vector $x$ contains the pixels in raster scan order-stacking the top row of the image, then the second row, etc. Specification of the model consists of specifying both the dimension of the parameter vector $\beta$ and the neighborhood system $\delta_{i}$. In this case, two models are distinct if their parameter spaces have different dimensions, or they are associated with different neighborhood systems or both. To this

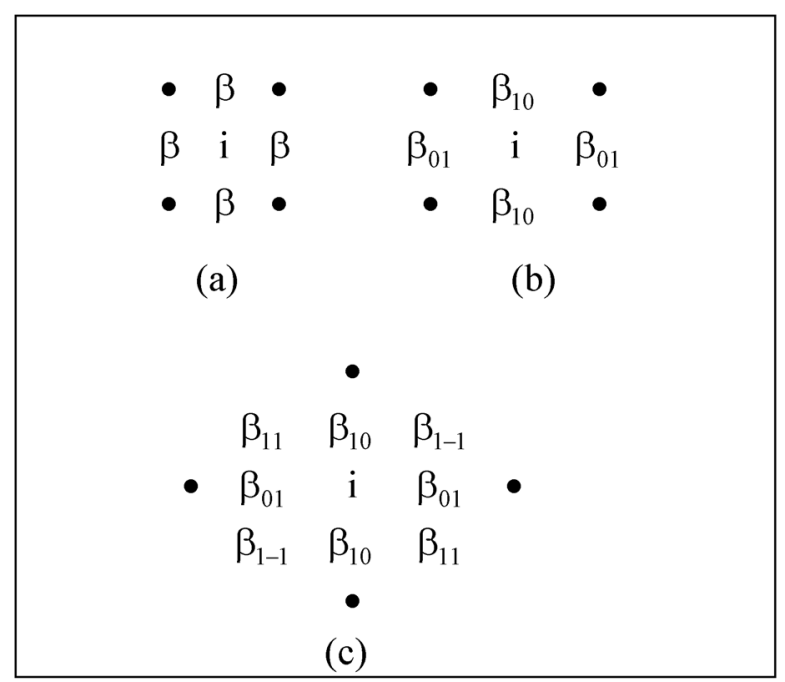

Fig. 1. Neighborhood systems and spatial interaction parameters of a GMRF. purpose, Figure 1 serves to illustrate the model selection problem.

As it can be seen, the model depicted in Figure 1(a) denotes a first order isotropic GMRF with single parameter $\beta$. In this case, each site has four neighbours. For the model in Figure $1(b)$, every site has again four nearest neighbours; however, different parameters $\beta_{01}$ and $\beta_{10}$ are used for horizontal and vertical pairs interactions. Finally, Figure 1(c) denotes a second order GMRF where the neighbourhood system has an expanded graph which includes also the diagonal elements (and parameters) in the southeast and northeast directions. Thus, since the impact of a specification error onto the quality of standard estimation procedures is serious [10], in this case automatic selection criteria can also be helpful when different models seem viable to fit the data.

\subsection{The ALSE Estimator}

In this section we present an estimator of model parameters that can be used to drive the automatic model order selection phase when the data are corrupted by a measurement error.

For the sake of simplicity, we describe the method for the temporal case, but the extension to the spatial case is straightforward [9].

Provided the order, $m$, of the process is known, the exact log-likelihood function for the process in (2), can be accomplished numerically to obtain the maximum likelihood estimates of the parameters. In contrast, conditional on the first $m$ observations, the log-likelihood assumes a simpler form and it is easy to show [12] that the conditional maximum likelihood estimates

$$
\hat{\phi}=\left(\sum_{t} \tilde{x}_{t}^{\prime} \tilde{x}_{t}\right)^{-1}\left(\sum_{t} \tilde{x}_{t} x_{t}\right)
$$

can be obtained as an OLS regression of $x_{t}$ on its own $m$ lagged values placed in the vector $\tilde{x}_{t}$. Notice that for the GMRF model the least squares estimator is better known as maximum Pseudo-Likelihood estimator [3]. However, as we have shown in the preceding section for the noisy case, a crude and direct application of this estimator leads to biased results. In order to obtain an estimator which takes care of the noise, let us specify $\tilde{y}_{t}=\tilde{x}_{t}+\tilde{\eta}_{t}$, where $\tilde{\eta}_{t}$ represents the set of the lagged variables for the 
observational noise. In this case, considering that

$$
\begin{aligned}
\sum_{t} \tilde{y}_{t}^{\prime} \tilde{y}_{t} & =\sum_{t}\left(\tilde{x}_{t}+\tilde{\eta}_{t}\right)^{\prime}\left(\tilde{x}_{t}+\tilde{\eta}_{t}\right) \\
& =\sum_{t}\left(\tilde{x}_{t}^{\prime} \tilde{x}_{t}\right)+2 \sum_{t}\left(\tilde{x}_{t}^{\prime} \tilde{\eta}_{t}\right)+\sum_{t}\left(\tilde{\eta}_{t}^{\prime} \tilde{\eta}_{t}\right)
\end{aligned}
$$

we can use the properties of the moment estimators to show that

$$
\begin{aligned}
& \frac{1}{n} \sum_{t=1}\left(\tilde{x}_{t}^{\prime} \tilde{\eta}_{t}\right)=O_{p}\left(n^{-1 / 2}\right) \\
& \frac{1}{n} \sum_{t}\left(\tilde{\eta}_{t}^{\prime} \tilde{\eta}_{t}\right)=\sigma_{\eta}^{2} I_{m}+O_{p}\left(n^{-1 / 2}\right)
\end{aligned}
$$

where $I_{m}$ is the $(m \times m)$ identity matrix. Finally, given that

$$
\frac{1}{n} \sum_{t}\left(\tilde{y}_{t} y_{t}\right)=\frac{1}{n} \sum_{t=1}\left(\tilde{x}_{t}^{\prime} \tilde{x}_{t}\right)+\sigma_{\eta}^{2} I_{m}+O_{p}\left(n^{-1 / 2}\right)
$$

it follows that substituting the large sample approximations suggested by (6) and (7) into (5), we have the Adjusted Least Square Estimator $(A L S E)$ based on the noisy series

$$
\tilde{\phi}=\left(\sum_{t} \tilde{y}_{t}^{\prime} \tilde{y}_{t}-n \sigma_{\eta}^{2} I_{m}\right)^{-1}\left(\sum_{t} \tilde{y}_{t} y_{t}\right)
$$

Furthermore, since

$$
\frac{1}{n} \sum_{t} y_{t}^{2}-\frac{1}{n} \sum_{t} x_{t}^{2}=\sigma_{\eta}^{2}+O_{p}\left(n^{-1 / 2}\right)
$$

it is straightforward to show that the Adjusted estimate of the residual variance is

$$
\tilde{\sigma}_{\epsilon}^{2}=\frac{1}{n} \sum_{t}\left(y_{t}-\tilde{\phi}^{\prime} \tilde{y}_{t}\right)^{2}-\sigma_{\eta}^{2}\left(1+\tilde{\phi}^{\prime} \tilde{\phi}\right)
$$

where $\sigma_{\eta}^{2}\left(1+\tilde{\phi}^{\prime} \tilde{\phi}\right)$ is the adjustment which takes care of the noise.

Finally, notice that the estimator is weakly consistent for $\phi$. In fact, from model assumptions, $(6),(7)$ and (8), it follows that

$$
\tilde{\phi}=\hat{\phi}+O_{p}\left(n^{-1 / 2}\right)
$$

However, it is well known that the OLS is a consistent estimator for $\phi$ under the noise free model, i.e.

$$
\hat{\phi}=\phi+o_{p}(1)
$$

hence, from (10) we have that

$$
\tilde{\phi}=\phi+o_{p}(1)
$$

as required. Thus, this results mean that the estimator is appropriate for large series.

\section{Model Order Selection Using Information Criteria}

Model identification involving order- selection criteria is usually based on the minimization of a loss function of the following form

$$
H(\cdot)+P(n, m)
$$

where $P(n, m)$ is a nonnegative random variable depending directly on sample size $n$ and the number of estimated parameters $m$ of the candidate model. In practice, $P(n, m)$ measures the complexity of the candidate model and serves as a penalty term for overfitting. On the other hand, $H(\cdot)$ is a measure of goodness-of-fit of the candidate model to the data and is dependent on the sample estimator in (9) of the residual variance $\tilde{\sigma}_{\epsilon}^{2}$ (or the conditional variance, $\tilde{\tau}^{2}$, in the spatial case).

According to (11) a wide range of criteria have been proposed in literature to estimate the expected Kullback-Leibler information. They can be categorized in asymptotically efficient or consistent criteria. For example, those that are asymptotically efficient and frequently used for autoregressive models selection are AIC [1], AICC [14] and CAT [19]. Those that are consistent, in the sense of picking the true order of the system with probability one asymptotically, are BIC [2], SIC [20] and HQ [13]. For a review of these methods and others which have not been mentioned here, see [4].

The model order selection approaches investigated here, are all based on the ALSE estimator with some form of penalty term attached. In particular, to deal with small and large samples, the following two different methods are implemented

$$
\begin{aligned}
& \mathrm{AICC}=n \log \tilde{\sigma}_{\epsilon}^{2}+2 n(m+1) /(n-m-2) \\
& \mathrm{SIC}=n \log \tilde{\sigma}_{\epsilon}^{2}+m \log n
\end{aligned}
$$




\section{Simulation Results}

For experimental purposes we have conducted some simulations to investigate, both in time and in space, the performance of the AICC and SIC statistics in the noisy case.

In particular, for the time series case, we have applied the information criteria to several simulated autoregressive processes. However, to save space, we limit here our discussion to the following second order autoregressive process

$$
X_{t}=0.99 X_{t-1}-0.8 X_{t-2}+\varepsilon_{t}
$$

where $\varepsilon_{t} \sim N(0,1)$. We have generated 500 realizations with two different sample sizes: $n=35$ and $n=100$. To each realization we have then added a white noise measurement error with variances $\sigma_{\eta}^{2}$ equal to 0.4 and 0.6. Finally, for each realization, parameters and residual variance of the candidate models were estimated by the $A L S E$ estimator and the criteria expressed in (12) and (13) were used to select from among the candidate models. Out of the 500 realizations the percentages of the model orders selected were tabulated for each criterion, sample size and model. The same simulation design was also followed for the GMRF model. In particular, we have simulated 200 zero-mean GMRFs images. In each set of simulation the images consist of $(20 \times 20)$, $(32 \times 32)$ and $(128 \times 128)$ pixels. Furthermore, as shown in Figure 1, we have simulated a first order isotropic process (IS-M) with parameter $\beta=0.35$; a first order homogeneous process $(\mathrm{FO}-\mathrm{M})$ with parameters $\beta_{01}=0.15$ and $\beta_{10}=0.3$ and, finally, a second order model (SO-M) with parameters $\beta_{01}=0.2, \beta_{10}=0$, $\beta_{11}=0$ and $\beta_{1-1}=0.2$. In all cases the conditional variance $\tau^{2}$ and the noise variance $\sigma_{\eta}^{2}$ were fixed, respectively, at 100 and 30 to obtain a SNR close to 4.5. The Tables 2 and 3 describe the model order selection results for the temporal and spatial cases. As it can be seen from Table 2, AICC is most successful at small $n$, whereas SIC is most successful at large $n$. However, even if the highest frequencies are observed in correspondence of the correct model order, the difficulty of selecting the true order as the SNR decreases is evident. As regards the spatial context, the consistent property of the SIC statistic, that seems to pick the exact model

\begin{tabular}{|c|c|c|}
\hline & \multicolumn{2}{|c|}{$\sigma_{\eta}^{2}=0.4$} \\
\hline$O S-A I C C$ & $n=35$ & $n=100$ \\
\hline 1 & $10.2(0.6)$ & $0.6(0)$ \\
\hline 2 & $64.8(82.6)$ & $60.8(78.6)$ \\
\hline $3-6$ & $25(16.8)$ & $38.6(20.2)$ \\
\hline $7-10$ & $0(0)$ & $0(1.2)$ \\
\hline$O S-S I C$ & \multicolumn{2}{|r|}{$n=100$} \\
\hline 1 & $16.2(1)$ & $0(0)$ \\
\hline 2 & $64.2(91.6)$ & $67.4(95)$ \\
\hline $3-6$ & $19.6(7.4)$ & $31.6(5)$ \\
\hline \multirow[t]{2}{*}{$7-10$} & $0(0)$ & $0(0)$ \\
\hline & \multicolumn{2}{|c|}{$\sigma_{\eta}^{2}=0.6$} \\
\hline$O S-A I C C$ & \multicolumn{2}{|r|}{$n=100$} \\
\hline 1 & $26(13.4)$ & $3.2(4.8)$ \\
\hline 2 & $47.8(69.6)$ & $54(70.2)$ \\
\hline $3-6$ & $26.2(17)$ & $42.8(24.2)$ \\
\hline $7-10$ & $0(0)$ & $0(0.8)$ \\
\hline$O S-S I C$ & \multicolumn{2}{|r|}{$n=100$} \\
\hline 1 & $35(6.1)$ & $1.8(0.8)$ \\
\hline 2 & $43.6(85.4)$ & $58(59.4)$ \\
\hline $3-6$ & $21.4(8.5)$ & $40.2(39.6)$ \\
\hline $7-10$ & $0(0)$ & $0(0)$ \\
\hline
\end{tabular}

Table 2. The percentage of the order selected (OS) by AICC and SIC criteria in 500 realizations of the AR(2) process with measurement error variance equal to 0.4 and 0.6. In brackets are the percentage of the order selected for the noise-free $\operatorname{AR}(2)$ process.

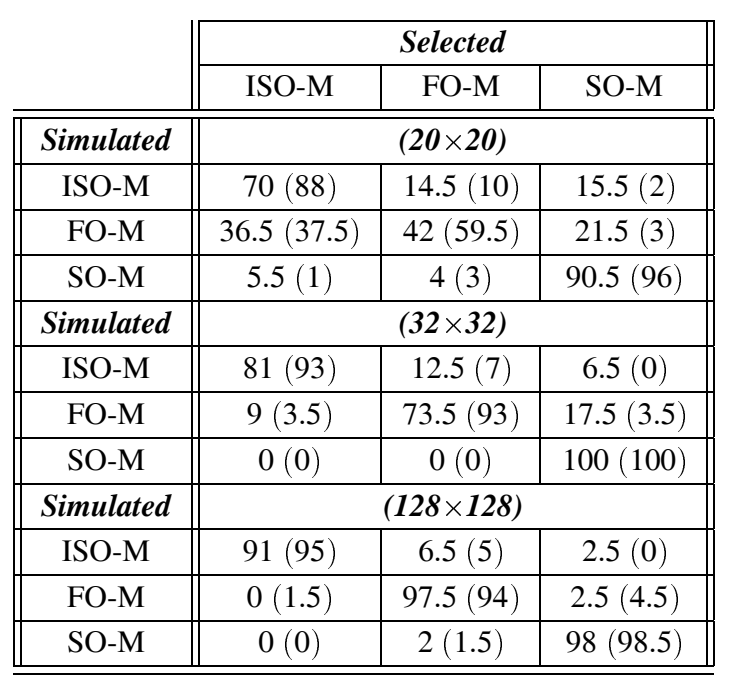

Table 3. The percentage of the order selected (OS) by the SIC criterion in 200 realizations of the GMRF. In brackets are the percentage of the order selected for the noise-free GMRF 
as the image size becomes larger is also evident. However, a point worth noting is that although an image of size $(20 \times 20)$ has 400 observations, multidirectional dependence structure of the spatial data complicates the identification phase. In fact, for the first order GMRF, there are $30 \%$ and $58 \%$ of wrong model selections, for the isotropic and homogeneous case, respectively.

\section{Conclusions}

We conclude this paper with some considerations. In Section 2.3 we have proposed an estimator of autoregressive model parameters that can be used in the automatic model selection phase when the data are corrupted by a measurement error. Because of its consistency, it can be used when huge data sets are available. In fact, with respect to the minimization of the exact maximum likelihood (EML) function the $A L S E$ estimator can be calculated quickly at the low computational cost of $O(n)$ steps. In the spatial and spatio-temporal context, Coli and Ippoliti [7] showed that under general boundary conditions EML can be calculated at the computational cost of $O\left(n^{2}\right)$ steps; however, for images of dimensions $(128 \times 128)$ or larger, the algorithm is computationally slow. Thus, the choice between EML and ALSE regards a trade-off between simplicity and efficiency.

In all the simulations $\sigma_{\eta}^{2}$ was treated as fixed and known. However, several techniques may be considered to obtain very good estimates. For example, in the field of wavelets, the $M A D$ estimator [8], gives robust estimates at the low cost of $O(n)$ operations. Additional techniques are also described in [15].

\section{References}

[1] AKAIKE, H., A new look at the statistical model identification, IEEE Trans. Automat. Control 1974; 19 , pp. 716-723.

[2] AKAIKE, H., Time series analysis and control through parametric models, in Findley, D. edition, Applied Time Series Analysis, Academic Press, New York, 1974.

[3] BESAG J., Efficiency of pseudolikelihood estimation for simple Gaussian fields, Biometrika 1977; 64, pp. 616-618.
[4] BHANSALI, R.J. Order selection for linear time series models: a review, in T. Subba Rao editor, Developments in Time Series Analysis, Chapman \& Hall, 1993, pp. 50-66.

[5] Box, G.E.P. AND Jenkins, G.M., Time Series Analysis: Forecasting and Control, 2nd Edition, San Francisco, 1976.

[6] Cressie N.A.C., Statistics for Spatial Data, Wiley, New York, 1993.

[7] Coli, M. AND IPPOLITI, L., Maximum Likelihood Estimation of Noisy Gaussian Markov Random Fields, in Glavinic, V., Hljuz Dobric, V. and Simic, D. editors, Proceeding $24^{\text {th }}$ International Conference on Information Technology Interfaces ITI 2002; Cavtat, Croatia, June 24-27, 2002.

[8] Donoho D.L., Johnstone I.M., Adapting to unknown smoothness via wavelet shrinkage, JASA 1995; 90:1200-24.

[9] Dryden I., Ippoliti L., Romagnoli L., Adjusted Maximum Likelihood and Pseudo-Likelihood Estimation for Noisy Gaussian Markov Random Fields, Journal of Computational and Graphical Statistics $2002 ; 11(2)$.

[10] GRIFFITH, D.A. AND LAGONA, F., On the quality of likelihood-based estimators in spatial autoregressive models when data dependence structure is misspecified, Journal of Statistical Planning and Inference 1998, 69, pp. 153-174.

[11] GUYON, X. AND YAO, J.F., On the underfitting and overfitting sets of models chosen by order criteria, Journal of Multivariate Analysis 1999; 70, pp. 221-249.

[12] Hamilton, J.D., Time Series Analysis, Princeton University Press, 1994.

[13] Hannan, E.J. AND QuinN, B.G., The determination of the order of an autoregression, J.R.S.S. 1979; B41, pp. 190-195.

[14] Hurvich, C.M. AND Tsai, C.L., Regression and time series model selection in small samples, Biometrika 1989; 76, pp. 297-307.

[15] IPPOLITI, L., RomagnOLI, L. AND FonTANELla L., A Noise Estimation Method for Corrupted Correlated Data, Technical Report DMQTE 2/2003, University of Pescara-Chieti.

[16] Ji, C. AND SEYMOUR, L., A consistent model selection procedure for Markov random fields based on a penalized pseudolikelihood, Annals of Applied Probability 1998; 6, pp. 423-443.

[17] LÜTKEPOHL, H., Comparison of criteria for estimating the order of a vector autoregressive process, Journal of Time Series Analysis 1985, 6, pp. 35-52.

[18] NewBold, P., Some recent developments in time series analysis, III. Int. Statist. Review 1988; 56, pp. 17-29. 
[19] PARZEN, E., Some recent advances in time series modelling and prediction problems, IEEE Trans. Auto. Control 1974; AC-19.

[20] SCHWARZ, G., Estimating the dimension of a model, Ann. Statist., 1978, 8, pp. 461-464.

Received: June, 2003

Accepted: September, 2003

Contact address:

Mauro Coli, Lara Fontanella, Luigi Ippoliti Department of Quantitative Method and Economic Theory University "G. d'Annunzio", Chiet

Viale Pindaro 42 65127 Pescara, Italy e-mail: coli@dmqte.unich.it

lf ontan@dmqte.unich.it

ippoliti@dmqte.unich.it

MAURo COLI is professor of statistics at the Department of Quantitative Methods and Economic Theory, University G. d'Annunzio (Chieti), where he is currently the coordinator of the Ph.D. courses in statistics. His main research interest includes spatial and spatio-temporal statistics with applications on social economic and environmental problems. $\mathrm{He}$ is a referee for the following journals: Statistica, Journal of Italian Statistical Society, Statistica Applicata; Ricerca Operativa.

LARA FONTANELLA received the Ph.D degree in statistics from the University G. d'Annunzio of Chieti in 2001 and a postdoctoral research fellowship from 2001 to 2002. She is a member of the Department of Quantitative Methods and Economic Theory where she is currently a teaching and research assistant. Her research interests are in spatial and spatio-temporal geostatistical models with particular consideration to environmental phenomena.

LUIGI IPPOLITI received the Ph.D degree in statistics from the University G. d'Annunzio of Chieti in 2000. Since 1995 he has been working at the Department of Quantitative Methods and Economic Theory where he is currently a researcher in statistics. In addition to spatial and spatio-temporal statistics, his current interest includes image analysis, multivariate analysis and computational statistics. 